\title{
ANALYSIS OF THE ELECTRICAL CHARACTERISTICS IN MULTIPHASE FLOW THROUGH THE WIRE-MESH SENSOR
}

\begin{abstract}
M. M. H. Cely,
H. F. P. Velasco,

and O. M. H. Rodriguez

Universidade de São Paulo Escola de

Engenharia de São Carlos

Laboratório de Engenharia Térmica e Fluidos

LETeF

Av. Trabalhador São Carlense, 400

CEP 13566-970, São Carlos, São Paulo, Brasil marlonhc@usp.br

\section{ABSTRACT}

Many studies on the characterization of electrical properties of multiphase fluid are found in the literature. One of the main motivations of these efforts has been the development of instrumentation for the measurement of volumetric fraction using electrical sensors. Although one can find a variety of instruments for that purpose, relatively few works in the open literature present studies on the best range of measurement frequency and its effect on permittivity models. An experimental and theoretical study is presented, where the best frequency to measure the volumetric fraction in two and three-phase mixtures is selected. Several permittivity models are applied to measure the volumetric fraction. The fluids used in the experiments were tap water, deionized water, mineral oil, isopropyl alcohol and hexane. Known volumes of fluids were mixed until obtaining a homogeneous mixture. The data were taken by a $1 \times 4$ wire-mesh sensor (WMS) immersed in the mixture. The WMS had a gap between planes of $1.4 \mathrm{~mm}$, the wires were $3 \mathrm{~mm}$ apart from each other and the diameter of the wires was of 0.2 $\mathrm{mm}$. The experimental system consisted of a generator, an oscilloscope and conditioning circuits (formed by operational amplifiers). A frequency scan was performed between $7000 \mathrm{~Hz}$ and $20 \mathrm{MHz}$ for each mixture. A total of 60 logarithmically spaced frequencies were applied.
\end{abstract}

Received: September 14, 2015

Revised: October 14, 2015

Accepted: November 09, 2015
Keywords: capacitive sensor, holdup, frequency, oil-water-Gas, volumetric fraction, wire-mesh

\section{NOMENCLATURE}

$V \quad$ Voltage (V)

$a, b$ Proportionality factors

\section{Greek symbols}

$\alpha \quad$ In-situ volumetric phase fraction (holdup) (-)

$\varepsilon \quad$ Relative permittivity (-)

\section{Subscripts}

$\begin{array}{ll}0 & \text { Oil } \\ w & \text { Water } \\ x & \text { Mixture } \\ i & \text { vertical index of the cross point } \\ j & \text { horizontal index of the cross point } \\ k & \text { temporal index } \\ t & \text { Time }\end{array}$

\section{INTRODUCTION}

The formation of water-in-crude oil emulsions during oil production can cause a substantial reduction of the production rates. This occurs due to the high effective viscosity of the emulsion that increases with water content towards the phase inversion point.

Therefore a particularly interesting but difficult measurement problem arises, namely how can the component fractions of an oil/gas/water mixture be reliably monitored in a hostile environment. Several techniques have been developed for the investigation of multiphase flows. Moreover the existing literature covers mainly the application of these techniques in gas-liquid flows. In liquid-liquid flows, (Angeli and Hewitt 2000) and (Lovick and Angeli 2004) used a high-frequency needle probe to obtain the phase distribution of oil and water over the pipe crosssection. Although images of oil fractions distribution were generated, they show only time-averaged data. (Zhao and Lucas 2011) have applied dual-sensor conductivity probes to generate local oil volume fractions distributions as well as velocity distributions in oil-in-water flows. The developed probe was capable to generate images of the flow, but with only limited spatial resolution. These authors also applied electrical resistance tomography (ERT) to characterize oil-water flow. (Li et al 2005) have also used ERT to measure oil fraction distributions. (Hasan and Azzopardi 2007) have employed electrical capacitance tomography (ECT) to investigate stratifying kerosene-water flow. However, electrical tomography's techniques are known to produce low spatial resolution images.

The WMS was first described by (I.D. Johnson, 1987) as a sensor for measuring the fraction of water in oil, based on fluid conductivity. Prasser et al. (Prasser, Böttger, and Zschau 1998) ensured the elimination of crosstalk between the electrodes. It 
was developed for application in nuclear power plants, in which water-steam flow is subjected to high temperatures and pressures. From there on the WMS is expanding its use in different areas. Recently, Da Silva (Da Silva, Schleicher, and Hampel 2007) developed a WMS based on the permittivity of the fluid, which expands the range of substances that can be identified. Also, the wire mesh has been replaced by different shapes (Damsohn and Prasser 2009b), (Damsohn and Prasser 2009a), (Belt et al. 2010), (Höhne, Kliem, and Bieder 2006) , but the operating principle and the associated electronic circuits are still the same.

\section{THEORY}

A WMS consists of two or three planes of wires. The wires are parallel in each plane and rotated $90^{\circ}$ between planes, Fig. 1 . The distance between the planes is of few millimeters or less ( 0.35 to $3 \mathrm{~mm})$, while the distance between the wires of a plane is a bit higher $(0.5$ to $15 \mathrm{~mm})$. These planes of wires are placed in the pipe's cross-sectional area. The space between each cross point is filled with fluid. Through a process of switching, each transmitter wire is activated sequentially, i.e., it sends an electrical signal to the fluid. Then, the receiver wire gets a signal that contains the information that identifies the type of fluid at each active cross point. More details on the operating principles of the conductive and capacitive WMS can be found in (Damsohn and Prasser 2009b) and (Da Silva et al. 2007), respectively. When the sequence of switching for the entire set of transmitters is complete, one slice with the information of the type of fluid or phase fraction on the cross-sectional plane is available, within a time interval. The union of these slices creates a 3D image of the topology of the flow. Also, it is possible to determine several important parameters of the flow from this image.

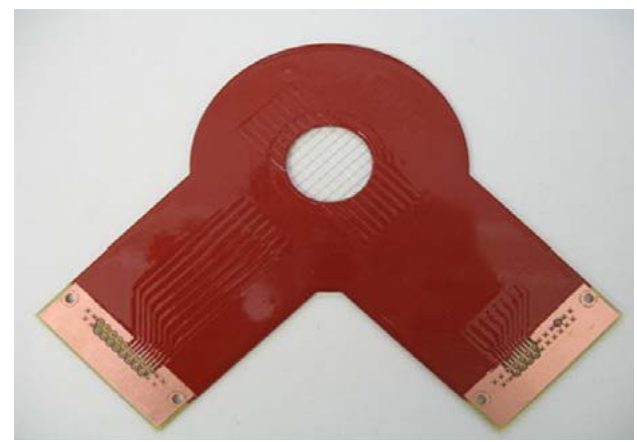

Figure 1 . Photo of a typical $8 \times 8$ WMS, schematic view of a typical wire-mesh sensor (WMS) located

to obtain a tomographic image in a pipeline.

The prototype employed in these experiments consists in a $1 \mathrm{x} 4$ wires grid ( $0.2 \mathrm{~mm}$ wire diameter) with axial spacing of the wire planes of $1.0 \mathrm{~mm}$ and 3 $\mathrm{mm}$ of wire separation on each plane of wires. The associated electronics measures the local electrical permittivity at all crossing points by successively applying a sinusoidal alternating voltage to each one of the sender electrodes at one wire plane and measuring in parallel the current flowing towards the receiver electrodes at the other wire plane see fig. 2 .


Figure 2. Sensor of wire electrodes (transmitter 1 and receiver 4 wires).

\section{NUMERICAL METHOD}

The main measurement that can be obtained by the WMS is the mean local conductivity or permittivity, which is directly measured without the need of any inverse reconstruction algorithm, compared to ECT (Electrical Capacitance Tomography). Afterwards, these are converted to local phase fraction (Damsohn and Prasser 2009a).

\section{A model of the electrical properties of the sensor and fluids}

The capacitive wire-mesh circuit delivers a voltage related with the relative permittivity according to

$$
V=a \cdot \ln \left(\varepsilon_{x}\right)+b
$$

where $V$ is the voltage, $\varepsilon_{x}$ is the relative permittivity of the mixture, and $a$ and $b$ are constants that depend on geometric factors and characteristics of the excitation signal. Thus, there is a variation in the measured values at each cross point for the same fluid. Therefore, it is necessary an adjustment or calibration process to compensate this variation. The adjustment procedure consists of measuring a substance of low permittivity and a known permittivity value $L$ (oil for $\varepsilon$ example, $\varepsilon_{o}=3.5$ ) covering the entire sensor. Thus, it is obtained a 
reference voltage data array denoted as $V_{L}$ :

$$
V_{L}(i, j)=\frac{1}{N_{t}} \sum_{k=0}^{N_{t}} V\left(\varepsilon_{L}, i, j, k\right)
$$

which is an raw data average over a range time, $k=$ $0, \ldots, N_{t}$. Here, $i$ and $j$ are the index of the cross point and $k$ is the time index. The procedure is repeated whit the sensor covered with another substance with higher permittivity, $\varepsilon_{H}$, providing $\varepsilon$ another reference voltage array denoted as $V_{H}$. Thus, applying (1) to the reference arrays $V_{L}$. and $V_{H}$. the constants a and $\mathrm{b}$ are calculated for each cross point as

$$
\begin{gathered}
\alpha(i, j)=\frac{V_{H}(i, j)-V_{L}(i, j)}{\ln \left(\varepsilon_{H}\right)-\ln \left(\varepsilon_{L}\right)} \\
b(i, j)=\frac{V_{L}(i, j) \ln \left(\varepsilon_{H}\right)-V_{H}(i, j) \ln \left(\varepsilon_{L}\right)}{\ln \left(\varepsilon_{H}\right)-\ln \left(\varepsilon_{L}\right)}
\end{gathered}
$$

Thus, the values of permittivity for each crossing point, $\varepsilon_{x}$, over the entire cross section can be determined by

$$
\varepsilon_{x}(i, j, k)=\exp \left(\frac{V(i, j, k)-b(i, j)}{a(i, j)}\right)
$$

\section{Relation Models (Permittivity models)}

To find the relationship between the relative permittivity obtained by the sensor $\varepsilon_{x}$ (Eq. (5)) and the in-situ phase fraction (holdup) $\alpha$, it is necessary to use a model that relates these two properties. There are several studies about estimating permittivity of mixtures, but most of them are focused on gas-liquid mixtures investigation, (Jaworek and Krupa 2010). (Karkkainen et al. 2000) and (Hao, T. Ed. 2005) present a summary of different models founded in the literature. Each model was designed for specific electrodes geometry and different distribution of the phases (flow pattern). For example, the Parallel Model represents a flow pattern where two immiscible phases form a circuit of virtual capacitors in parallel

$$
\alpha_{o}(i, j)=\frac{\varepsilon_{w}-\varepsilon_{x}}{\varepsilon_{w}-\varepsilon_{o}}
$$

If the configuration of the two phases forms a circuit with the virtual capacitors in series, the relation becomes

$$
\alpha_{o}(i, j)=\left(\frac{\varepsilon_{w}-\varepsilon_{x}}{\varepsilon_{w}-\varepsilon_{o}}\right)\left(\frac{\varepsilon_{o}}{\varepsilon_{x}}\right)
$$

\section{EXPERIMENTS}

Experiments were carried out in a becker de 250 $\mathrm{ml}$, fig. 3 , to the input signal generator was used $20 \mathrm{MHz}$ measuring the output signal by DSOX2014A Oscilloscope of $100 \mathrm{MHz} 4$ Channels connected to an Intel core i7 PC using labview 2012 software with which the output signals that allowed me to know the electrical characteristics (voltage gain) of each component is obtained.

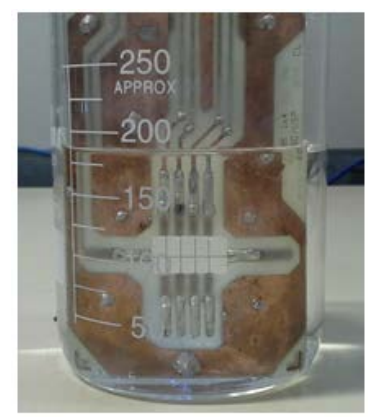

Figure 3. Experiments were carried out in a becker de $250 \mathrm{ml}$.

Mixes for the different oil water and gas were analyzed. Signal $V i$ were injected with a frequency sweep from $7000 \mathrm{~Hz}$ to $20 \mathrm{MHz}$ by Agilent oscilloscope. A total of 60 logarithmically spaced frequencies were applied. Errors were calculated and used to select the best measurement frequencies for each tested mixture.

\section{RESULTS AND DISCUSSION}

\section{Mixture Tap Water-Alcohol}

The response of the mixture of alcohol tap water shown in Figures 4 and 5. This can be seen as change the gain and phase with the water fraction and frequency changes. It can be observed in the gain response Figure 4 as water shows two plateaus were expected according to the theory. Due to the lower limit of frequencies alcohol shows only the highfrequency plateau, which shows that the lower plateau lies below this limit. The answer due to the gain-wide operational amplifier bandwidth product used is not yet visible.

The phase response is illustrated in Figure 5. One can see how the water at $7 \mathrm{kHz}$ response begins near $180^{\circ}$, moves to a minimum near $300 \mathrm{kHz}$ and then begins to return to $180^{\circ}$. But in the higher frequency decreasing back again, this time because the operational amplifier response. This coincides with anticipated by theory. In the case of alcohol, the 
stage at $7 \mathrm{kHz}$ is $238.5^{\circ}$, topping out at around 15.7 $\mathrm{kHz}$ and then to $180^{\circ}$. The same as in the case of water, at high frequencies the phase is dominated by the response of the operational amplifier and down. This behavior of alcohol confirms that on $7 \mathrm{kHz}$ still has not reached the low frequency plateau. In addition, as the response should be symmetrical around the maximum displacement frequency, 15.7 $\mathrm{kHz}$, it is concluded that the frequency which begins the plateau of low frequencies is more than a decade below. This corresponds to frequencies in the order of hundreds of $\mathrm{Hz}$.

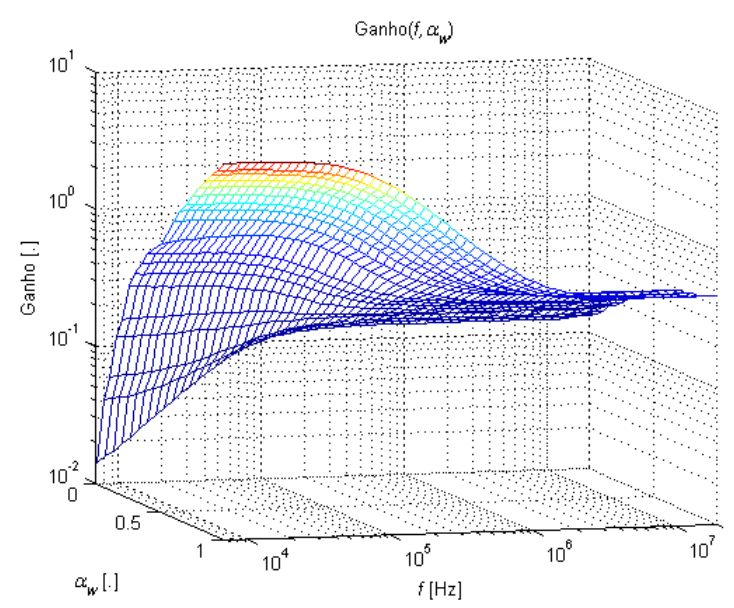

Figure 4. Frequency Response gain, mixture Tap Water Alcohol.

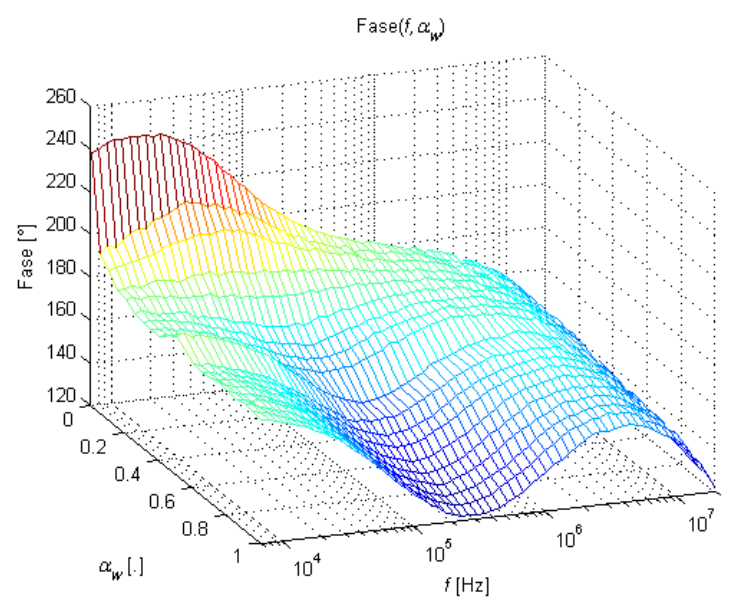

Figure 5. Frequency Response Phase, mixture Tap Water Alcohol.

It is noteworthy that the response phase can display the gain limit bandwidth operational amplifier, which is not possible for the gain in the frequency band analyzed Figure 4.

One can also see that the surfaces of the mix change smoothly and match very well with the theoretically expected values. This is because it is considered that the two fluids are highly polar and miscible allowing to obtain a stable emulsion.
The conductivity and permittivity for different fractions of alcohol are shown in Figures 6 and 7, respectively. These were obtained from measurements, and by measuring strategy calibration using as pure water and alcohol. A set of values is done through regression coefficients and R2 value is shown in Figures. In the case of the conductivity can be seen a highly nonlinear relationship. As for the permittivity is presented a linear relationship. The results are very similar to the experiment performed by Da Silva et al. (2007).

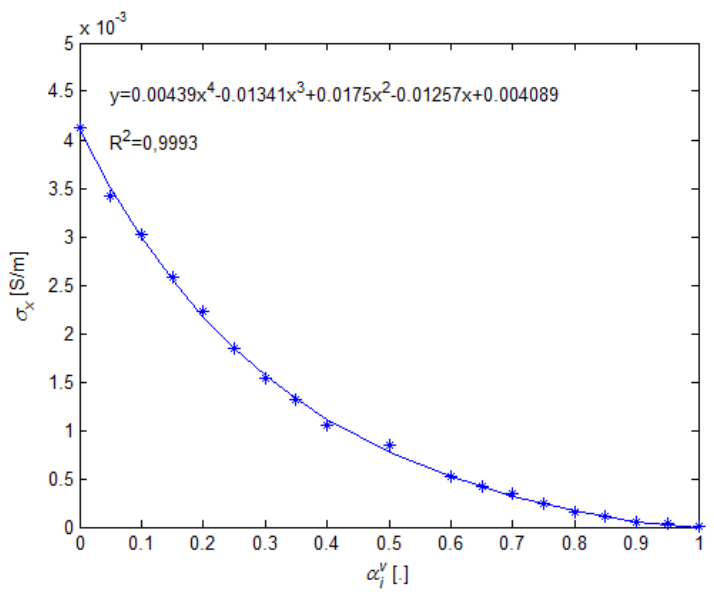

Figure 6. Conductivity of the mixture water tap alcohol for different fractions of alcohol, $f=204$ $\mathrm{kHz}$. Conductivity measurements $\left(^{*}\right)$ and adjust polynomial (line).

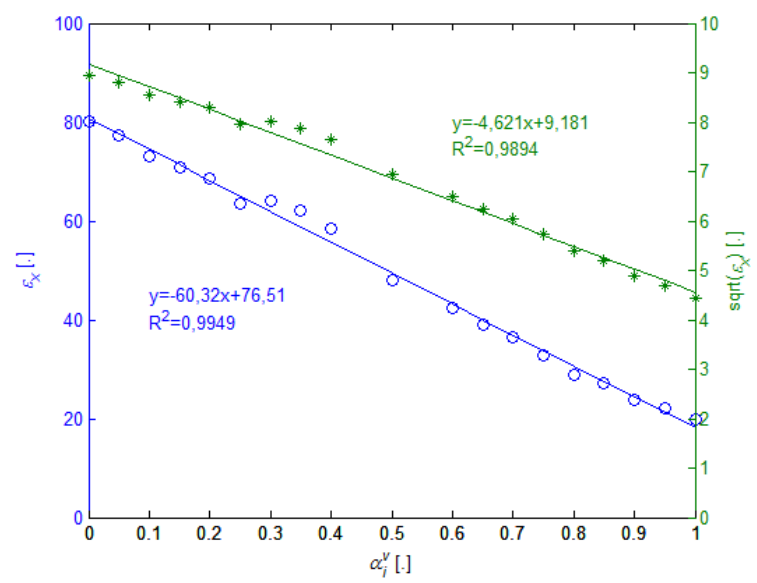

Figure 7. Permittivity of the mixture water tap alcohol for different fractions of alcohol, $f=204$ $\mathrm{kHz}$. Measurements of permittivity (o), square root of the permittivity $(*)$ and polynomial fit for the two (line).

\section{Mixture deionized water-Oil Mineral}

Figure 8 and 9, shows the frequency response for mixing deionized water mineral oil. This can be seen as oil displays only the high-frequency plateau is confirmed by electric phase response. Moreover, 
considering that the phase response for oil has not yet reached its peak and the symmetry that must have this, one can predict that the frequency for the start of the low frequency plateau is in the order of tens of $\mathrm{Hz}$ or lower. This coincides with the theoretically expected value. On the other hand, the deionized water has an almost completely flat response. This indicates that the frequency cut $\omega_{f}$ and $\omega_{x}$ are the same.

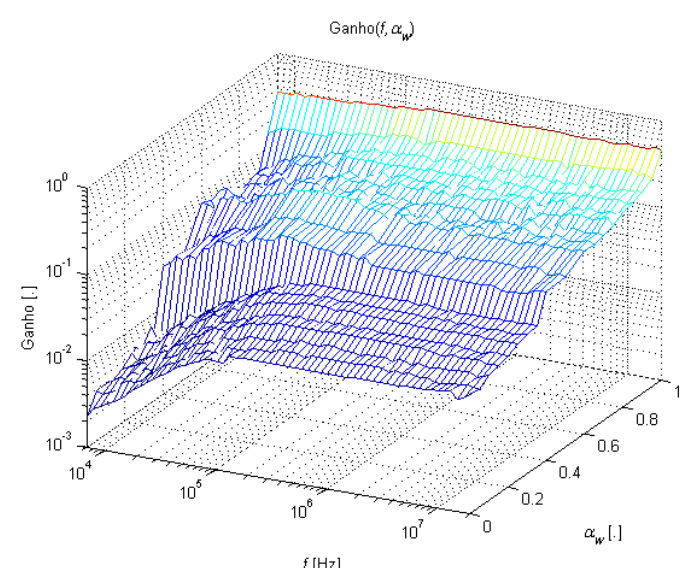

Figure 8. Frequency response for gain, mixture deionized water-Oil Mineral.

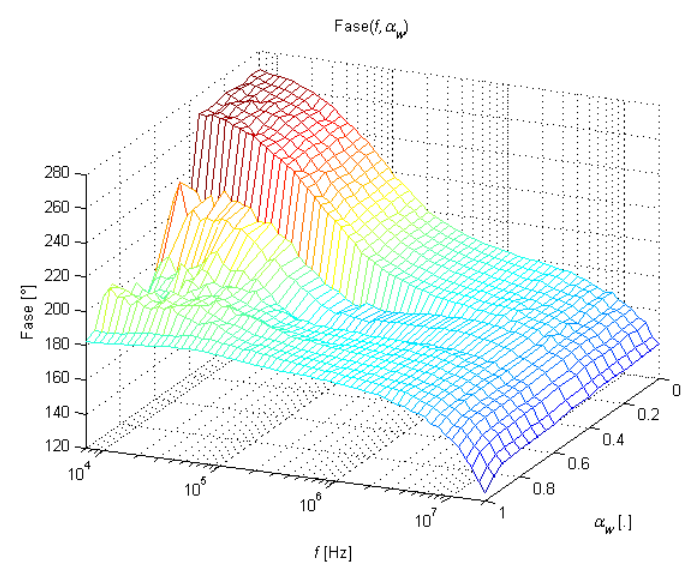

Figure 9. Frequency response for phase, mixture deionized water-Oil Mineral.

In Figure 8 highlights the rapidly changing gain values for $\left(0.35<\alpha_{w}<0.4\right)$. This change was not observed in this mixture and indicates a mixture does not self-consistent as Maxwell-Garnett. But none of the studied relationship models can satisfactorily explain the complex permittivity measurement. A comparison of the results obtained with some models relationship is shown in Figures 10 and 11.

The conductivity has a strong variation, Figure 10 , passing to adjust well at the extremes ( $\alpha_{w} \leq 0.35$ e $0.9 \leq \alpha_{\mathrm{w}}$ ) the models Maxwell w/O and Series have values close to the models Maxwell o $/ \mathrm{W}$ and parallel in the central strip $\left(0.4 \leq \alpha_{\mathrm{w}} \leq 0.6\right)$ and the remaining track does not fit into any of the models. For permittivity, Figure 11, one can see how the model the Maxwell-Garnett w/O coincides very well with the measurements for $\alpha_{w}<0.35$, as expected for this range fractions where it is expected that the oil is the continuous phase. In contrast, the Maxwell-Garnett type $\mathrm{o} / \mathrm{W}$ is very separated to the largest fraction, where water should be the continuous phase.

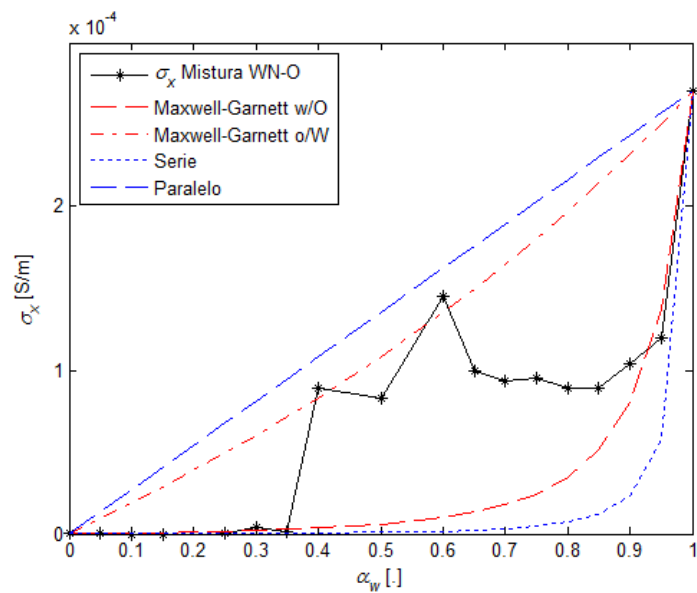

Figure 10. Conductivity mixing deionized water mineral oil to different fractions of water. The measurement was made for $\mathrm{f}=204 \mathrm{kHz}$.

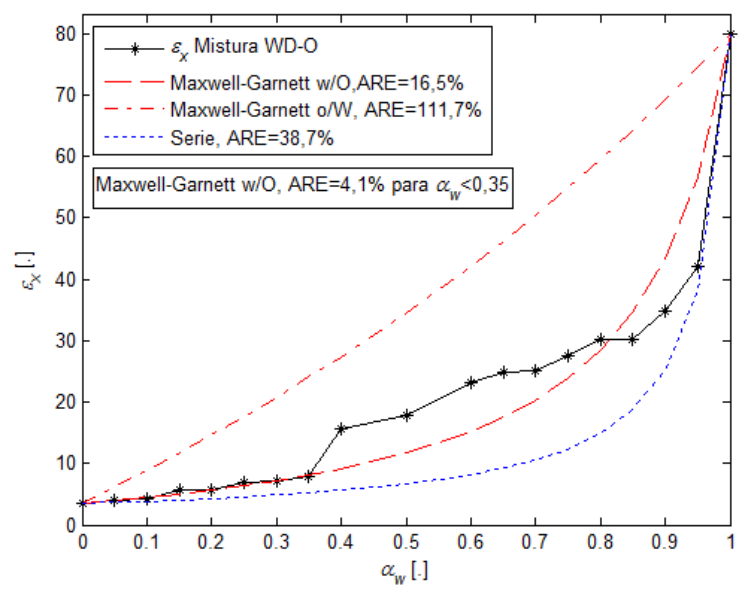

Figure 11. Permittivity mixing deionized water mineral oil to different fractions of water. The measurement was made for $\mathrm{f}=204 \mathrm{kHz}$.

\section{Mixture Tap Water-Mineral Oil}

The results of the frequency response for mixture tap water mineral oil are presented in Figures 12 and 13. Comparing this with the response of deionized water, mineral oil Figures 10 and 11, the response shows no rapid change for any particular fraction of water. On the other hand, it is observed that the behavior of surfaces measures is not as smooth as in mixtures listed above. It is considered that this is because the fluids are not miscible. 




Figure 12. Frequency response for gain, Mixture Tap Water Mineral-Oil.

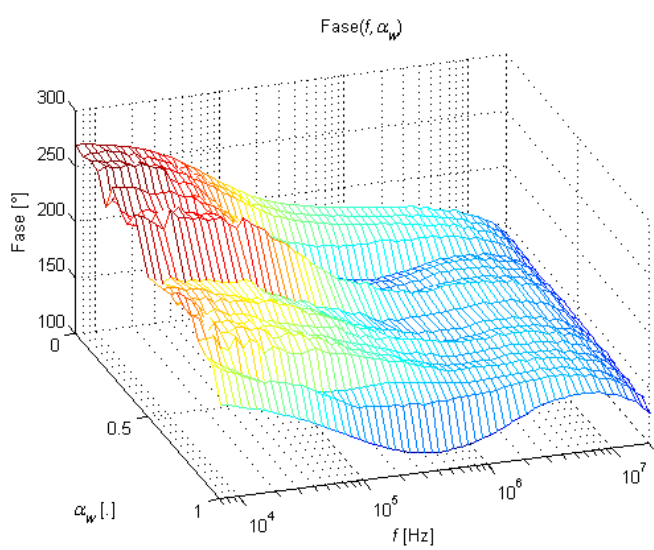

Figure 13. Frequency response for phase, mixture Tap Water Mineral-Oil.

The conductivity and permittivity for different water fractions are shown in Figures 14 and 15. In both cases, one can see how the relation is strongly non-linear, even more intensely than in the previous two mixtures. In particular, the conductivity is being dominated by oil in almost all the range of fractions Figure 14.

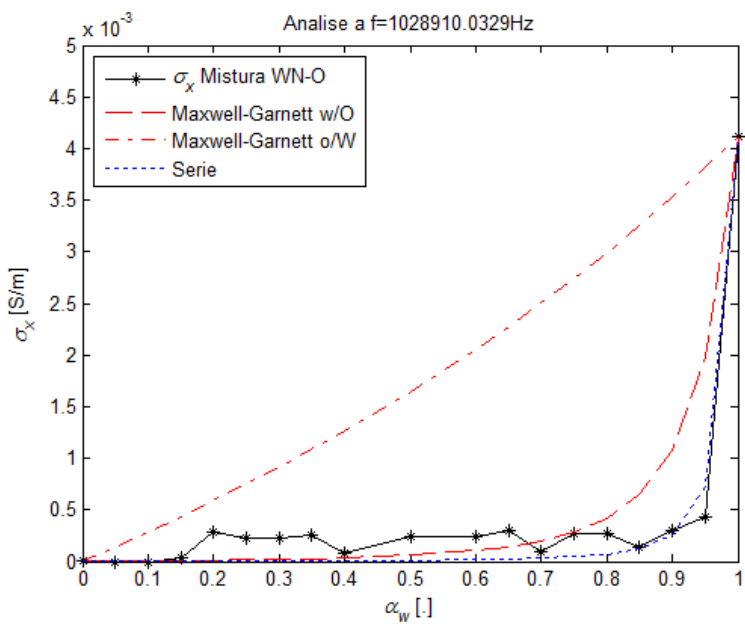

Figure 14. Conductivity mixing tap water for different oil fractions water, $\mathrm{f}=1.03 \mathrm{MHz}$.

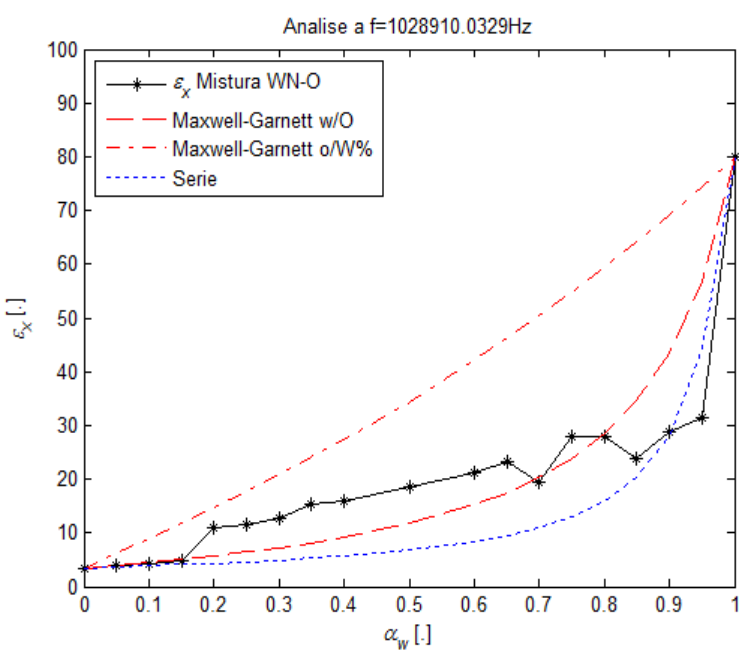

Figure 15. Permittivity mixing tap water for different oil fractions water, $f=1.03 \mathrm{MHz}$.

\section{Mixture Tap Water-Hexane}

In Figures 16 and 17, presents the frequency response for mixture tap water hexane. In the gain of the response, Figure 16, can be seen as hexane presents only the high-frequency plateau, being confirmed by phase response, Figure 17 . In the gain and phase responses can be seen that, unlike the water-alcohol mixture, has values of abrupt changes, especially at low frequencies, even greater than in the case of mineral oil and tap water. In this case also the two fluids are not miscible. Moreover, the mixtures had the largest bubbles of disperse phase, in comparison with the other mixtures, especially when the hexane was the dispersed phase. So, it is considered that the abrupt changes should be really that at the time of measurement is only had the presence of a phase in the crossover point.

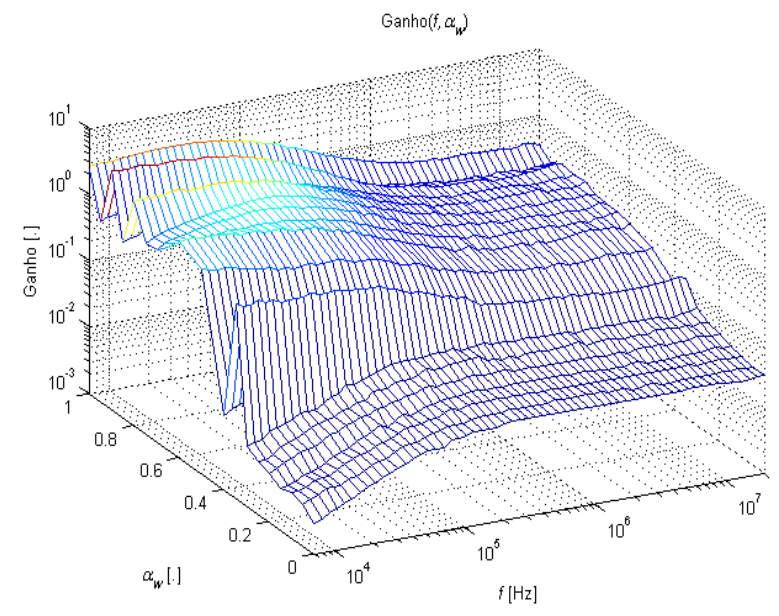

Figure 16. Frequency response for gain, mixture Tap Water-Hexane. 


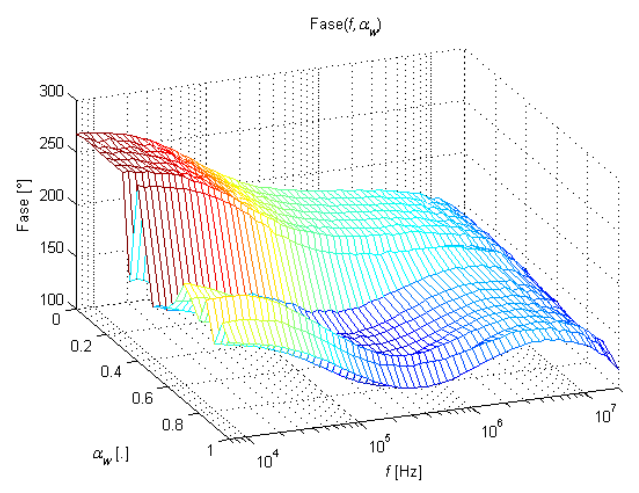

Figure 17. Frequency response for phase, Mixture Tap Water-Hexane.

\section{Mixture Oil-Hexane}

The frequency response for the hexane-mineral oil mixture was shown in Figures 18 and 19. It can be seen, Figure 18, the gain in the case of oil is slightly greater than that of hexane to greater than $1 \mathrm{MHz}$. At that frequency, the gain depends ideally only the relative permittivity; then the ratio of the gains should correspond the ratio of permissiveness. The phase response, Figure 19, shows no significant difference.



Figure 18. Frequency response for gain, mixture OilHexane.

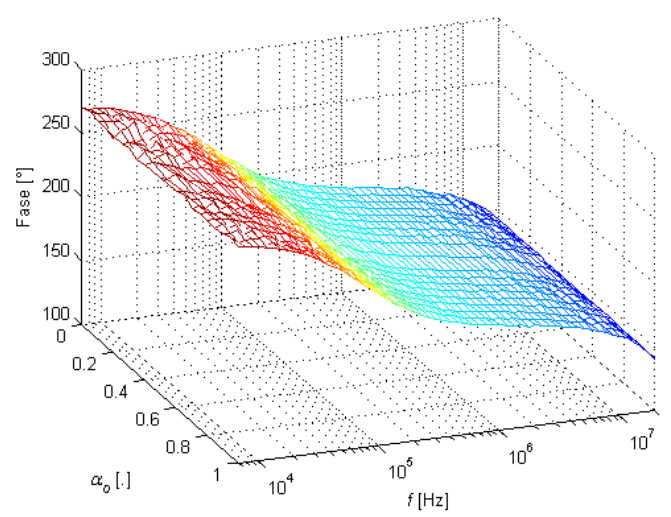

Figure 19. Frequency response for phase, mixture Oil-Hexane.

\section{CONCLUSIONS}

Each substance can be easily distinguished from each other either in the low frequency (resistive) plateau other in the high-frequency (capacitive) one. As previously described the conductance and capacitance of the measuring cell are linked respectively to the electrical conductivity and permittivity of the substance under test. The WMS was excited with a sine-wave signal with frequencies between $7 \mathrm{Kh}$ and $20 \mathrm{MHz}$, and the phase shift between the sensor response and reference signals was measured using a digital storage oscilloscope. It was demonstrated that the phase shift, which is nearly proportional to the void fraction, can be a measure for void fraction.

The measurement of amplitude is not a problem because the methodology used in capacitive type can be used. Now for the direct measurement of the phase, a simulation was made with different frequencies, as shown in the Figures. Experimentally, Jaworek and Krupa (2010) found that the maximum phase change is $14^{\circ}$ or $\Delta t=39$ ns with $1 \mathrm{MHz}$ and Da Silva, M. (2008) expect a variation of a few degrees with $5 \mathrm{MHz}$ in biphasic mixtures coincides with the simulations. However, these differences are not feasible for measurement. In the case of $\mathrm{f}=100 \mathrm{kHz}$ and $5 \mathrm{MHz}$ as can be seen for low fractions of the water phase contour lines are too close to each other. This means that you lose resolution and contrast in these cases, making very difficult the technique.

\section{ACKNOWLEDGEMENTS}

The authors acknowledge with gratitude the support of the companies STATOIL and PETROBRAS.

\section{REFERENCES}

Angeli, P., and Hewitt, G. F., 2000, Flow Structure in Horizontal Oil-Water Flow, International Journal Multiphase Flow, Vol. 26, pp. 1117-1140.

Belt, R. J., Van't Westende, J. M. C., Prasser, H. M., and Portela, L. M., 2010, Time and Spatially Resolved Measurements of Interfacial Waves in Vertical Annular Flow, International Journal of Multiphase Flow, Vol. 36, No. 7, pp. 570-587.

Damsohn, M., and Prasser, H. M., 2009, HighSpeed Liquid Film Sensor for Two-Phase Flows with High Spatial Resolution Based on Electrical Conductance, Flow Measurement and Instrumentation, Vol. 20, No. 1, pp. 1-14.

Damsohn, M., and Prasser, H. M., 2009b, HighSpeed Liquid Film Sensor with High Spatial Resolution, Measurement Science and Technology Vol. 20, No. 11, pp.1-9.

Da Silva, M. J., Schleicher, E., and Hampel, U., 2007, Capacitance Wire-Mesh Sensor for Fast Measurement of Phase Fraction Distributions, 
Measurement Science \& Technology, Vol. 18, No. 7, pp. 2245-2251.

Höhne, T., Kliem, S., and Bieder, U., 2006, Modeling of a Buoyancy-Driven Flow Experiment at the ROCOM Test Facility Using the CFD Codes CFX-5 and Trio U, Nuclear Engineering and Design, Vol. 236, No. 12, pp. 1309-1325.

Jaworek, A., and Krupa, A., 2010, Phase-Shift Detection for Capacitance Sensor Measuring Void Fraction in Two-Phase Flow, Sensors and Actuators A-Physical, Vol. 160, No. 2, pp. 78-86.

Johnson, I. D., 1987, Method and Apparatus for Measuring Water in Crude Oil, U.S. Patent 4664263.

Karkkainen, K. K., Sihvola, A. H., and Nikoskinen, K. I., 2000, Effective Permittivity of Mixtures Numerical Validation by the FDTD Method, IEEE Transactions on Geoscience and Remote Sensing, Vol. 38, No. 3, pp. 1303-1308.

Li, H., Wang, M., Wu, Y., Ma, Y., and Williams R., 2005, Measurement of Oil Volume Fraction and Velocity Distributions in Vertical Oil-in-Water Flows Using ERT and a Local Probe, Journal of Zhejiang University Science, Vol. 6, No. 12, pp. 1412-1415.

Lovick, J., and Angeli, P., 2004, Experimental Studies on the Dual Continuous flow Pattern in oilWater Flows, International Journal Multiphase Flow, Vol. 30, No. 2, pp. 139-157.

Prasser, H. M., Böttger, A., and Zschau, J., 1998, A New Electrode-Mesh Tomograph for GasLiquid Flows, Flow Measurement and Instrumentation, Vol. 9, No. 2, pp. 111-119.

Zhao, X., and Lucas, G. P., 2011, Use of a Novel Dual-Sensor Probe Array and Electrical Resistance Tomography for Characterization of the Mean and Time-Dependent Properties of Inclined, Bubbly Oil-in-Water Pipe Flows, Measurement Science and Technology, Vol. 22, No. 10, pp. 16-29. 